\title{
Intravenous ketamine plus midazolam is superior to intranasal midazolam for emergency paediatric procedural sedation
}

\author{
J P Acworth, D Purdie, R C Clark
}

\begin{abstract}
Objectives-This study compared intranasal midazolam (INM) with a combination of intravenous ketamine and intravenous midazolam (IVKM) for sedation of children requiring minor procedures in the emergency department.

Method-A single blinded randomised clinical trial was conducted in the emergency department of a major urban paediatric hospital. Subjects requiring sedation for minor procedures were randomised to receive either INM $(0.4 \mathrm{mg} / \mathrm{kg})$ or intravenous ketamine (1 $\mathrm{mg} / \mathrm{kg})$ plus intravenous midazolam $(0.1 \mathrm{mg} / \mathrm{kg})$. Physiological variables and two independent measures of sedation (Sedation Score and Visual Analogue Sedation Scale) were recorded before sedation and at regular intervals during the procedure and recovery period. Times to adequate level of sedation and to discharge were compared. Results-Fifty three patients were enrolled over a 10 month period. Sedation was sufficient to complete the procedures in all children receiving IVKM and in 24 of the 26 receiving INM. Onset of sedation was an average of 5.3 minutes quicker with IVKM than with INM $(95 \% \mathrm{CI} 3.2,7.4 \mathrm{~min}-$ utes, $\mathbf{p}<0.001)$. Children given INM were discharged an average of 19 minutes earlier than those given IVKM $(95 \%$ CI 4 , 33 minutes, $p=0.02$ ). Mean Sedation Scores and Visual Analogue Sedation Scale scores for the $\mathbf{3 0}$ minutes after drug administration were significantly better in children given IVKM compared with INM (2.4 and 1.8 versus 3.5 and 3.8 , respectively). Both doctors and parents were more satisfied with sedation by intravenous ketamine and midazolam.

Conclusions-Intravenous ketamine plus midazolam used in an appropriate setting by experienced personnel provides an excellent means of achieving sedation suitable for most non-painful minor procedures for children in the emergency department. This combination is superior to INM in terms of speed of onset and consistency of effect. INM delivered via aerosol spray has a more variable effect but may still be adequate for the completion of many of these procedures.

(Emerg Med f 2001;18:39-45)
\end{abstract}

Keywords: intranasal midazolam; intravenous ketamine; sedation; children
The provision of adequate analgesia and anxiolysis is an important role for emergency physicians. Despite increased awareness of this subject in the medical literature, inadequate attention to pain and anxiety in paediatric patients continues. Recent reviews ${ }^{1-5}$ have centred on safety issues in the delivery of sedative drugs in the non-operating room setting and highlight the need for controlled trials of different sedative protocols used in children in varied settings. Ketamine and midazolam are two agents commonly used for sedating children during medical procedures but despite their reported suitability for procedural sedation in the emergency department (ED) setting $^{145}$ their use remains erratic.

This study aimed to compare intranasal midazolam (INM) with a combination of intravenous ketamine plus intravenous midazolam (IVKM) for sedation of children requiring minor procedures, such as laceration repair or foreign body removal, in the ambulatory setting. A comparison of these methods in this particular setting has not previously been published. The ED in this study had existing protocols for these techniques and although INM was being used more frequently than IVKM, there were concerns regarding its reliability. We hypothesised that IVKM would more reliably produce a suitable level of sedation for minor procedures than INM and that both doctors and parents would be more satisfied with this method of sedation.

\section{Method}

STUDY DESIGN

This was a single blinded randomised controlled trial conducted in a major urban paediatric hospital with an ED seeing 19000 patients annually. The study was approved by the hospital's Ethics Committee and Clinical Drug Trial Subcommittee.

\section{ELIGIBILITY}

Children were eligible for the study if they were aged 6 months to 12 years, haemodynamically and neurologically stable, and needed a procedure likely to cause distress. The procedure had to either be non-painful or one in which the pain could be removed with local anaesthetic (table 1). Children with a history of an adverse reaction to midazolam or ketamine, psychiatric or behavioural disorder, risk of raised intracranial or intraocular pressure, thyroid disorder, porphyria, blocked nose, or who had been sedated within four hours of presentation were excluded from the study. Children 
Table 1 Baseline characteristics of treatment groups

\begin{tabular}{llll}
\hline Parameter & $I V K M^{*}$ & $I N M \dagger$ & $p \ddagger$ \\
\hline Age (years (SD)) & $4.6(2.9)$ & $3.5(2.0)$ & NS \\
Weight (kg (SD)) & $20.4(11.5)$ & $16.5(3.8)$ & NS \\
Male sex (\%) & $14 / 26(54)$ & $15 / 26(58)$ & NS \\
Indication for sedation & & & \\
$\quad$ Laceration repair (\%) & $14 / 26(54)$ & $19 / 26(73)$ & NS \\
$\quad$ Foreign body removal (\%) & $10 / 26(38)$ & $7 / 26(27)$ & NS \\
$\quad$ Other (\%) & $2 / 26(8)$ & $0 / 26(0)$ & NS \\
Lacerations & & & \\
$\quad$ Location (\% facial) & $9 / 14(64)$ & $13 / 19(68)$ & NS \\
$\quad$ Length (cm) & $2.8(2.3)$ & $3.5(2.1)$ & NS \\
Duration of procedure (min (SD)) & $14.2(10.9)$ & $16.4(13.8)$ & NS \\
Pulse rate (beat/min (SD)) & $103.2(17.8)$ & $102.7(14.2)$ & NS \\
Respiratory rate (min ${ }^{-1}$ (SD)) & $24.6(5.8)$ & $26.5(4.6)$ & 0.04 \\
Mean arterial pressure (mm Hg (SD)) & $79.8(8.5)$ & $75.1(11.0)$ & NS \\
Oxygen saturation (\% (SD)) & $98.0(0.8)$ & $97.7(1.0)$ & NS \\
Pre-procedure sedation score (SD) & $3.8(1.0)$ & $3.6(0.8)$ & NS \\
Pre-procedure VASS score (SD) & $4.3(3.2)$ & $5.5(2.5)$ & NS \\
\hline
\end{tabular}

*Intravenous ketamine plus midazolam group. †Intranasal midazolam group. $\neq \mathrm{p}$ Value $>0.05$ considered not statistically significant (NS).

\begin{tabular}{|l}
\hline Fig 1 Sedation Score \\
1 Unconscious-asleep, does not respond to minor motor stimulation \\
2 Drowsy-drowsy, may close eyes but responds to minor motor stimulation \\
3 Normal state-calm, sitting/lying comfortably with eyes open \\
4 Anxious-looks frightened but not clinging to parent, may whimper but not crying \\
5 Very agitated-crying, struggling or clinging to parent
\end{tabular}

Figure 1 Sedation Score.

were fasted while in the department awaiting the procedure but no minimum duration of starvation was required before drug administration. Eligible children presenting to the ED between 8 am and $10 \mathrm{pm}$ from March 1998 to January 1999 and for whom written informed consent was obtained were recruited into the study.

RANDOMISATION AND BLINDING

Subjects were randomised using computer randomised allocations sealed in opaque envelopes to receive either intranasal midazolam $0.4 \mathrm{mg} / \mathrm{kg}$ (maximum $10 \mathrm{mg}$ ) or intravenous ketamine $1 \mathrm{mg} / \mathrm{kg}$ (no maximum) plus intravenous midazolam $0.1 \mathrm{mg} / \mathrm{kg}$ (maximum 5 $\mathrm{mg}$ ). The doctor and nurse responsible for scoring the child's sedation level were not present during the drug administration and were blinded to each subject's allocation by the use of a dummy armboard applied to children receiving the intranasal preparation. To evaluate the possibility of bias from lack of blinding, after each of the final 20 cases the scorers were asked to offer an opinion about which medication they thought the child had been given.

Patient is alert
Observations are normal
Speech/communication is appropriate for age
Play is appropriate for age
No nystagmus
Able to walk unaided (if age appropriate) without ataxia

Figure 2 Discharge criteria.

INTERVENTIONS

The procedures were performed in a room equipped with resuscitation equipment and a doctor skilled in advanced airway management administered the sedation. In the INM group, the parenteral form of midazolam was used undiluted $(5 \mathrm{mg} / \mathrm{ml})$ and the dose was given via a metered dose spray device ${ }^{6}$ delivering 0.2 $\mathrm{ml} /$ spray. Half the dose was delivered into each nostril while the parent held the child. Children in the IVKM group received intravenous midazolam $(5 \mathrm{mg} / \mathrm{ml}$ solution diluted to $1 \mathrm{mg} / \mathrm{ml}$ with $0.9 \%$ saline) followed by intravenous ketamine $(100 \mathrm{mg} / \mathrm{ml}$ solution diluted to $10 \mathrm{mg} / \mathrm{ml}$ with $0.9 \%$ saline) given over one minute. If adequate conditions for the procedure were not attained within $20 \mathrm{~min}$ utes, "top up" doses of $0.5 \mathrm{mg} / \mathrm{kg}$ of intravenous ketamine were available. All lacerations were infiltrated with $1 \%$ lignocaine (lidocaine). After the procedure, children were nursed in a quiet area until recovery.

\section{OUTCOME MEASURES}

Measured physiological variables included pulse rate $(P R)$, respiratory rate $(R R)$, mean arterial blood pressure (MAP) and oxygen saturation measured by pulse oximetry $\left(\mathrm{O}_{2}\right.$ Sat). Level of sedation was assessed using the Sedation Score, which is a 5 point analogue scale (fig 1) previously validated for the assessment of procedure related anxiety in children. ${ }^{7}$ The Sedation Score and physiological variables were recorded before drug administration, at five minute intervals until the procedure ended, then at 10 minute intervals until discharge by the nurse observer. Procedures started once the child's Sedation Score was 3 or less. The Visual Analogue Sedation Scale (VASS) is a visual analogue scale developed specifically for this study. The doctor performing the procedure was asked to rate the child's level of sedation by indicating a point on a 10 $\mathrm{cm}$ line between the labels "quiet and still or asleep" (at $0 \mathrm{~cm}$ ) and "uncontrolled crying and

Table 2 Outcome characteristics of treatment groups

\begin{tabular}{|c|c|c|c|c|}
\hline Parameter (SD) & $I V K M^{*}$ & INMt & $p \neq$ & Mean difference $(95 \% \mathrm{CI})$ \\
\hline Time to onset of sedation (min) & $2.0(0.0)$ & $7.31(5.26)$ & $<0.001$ & $5.3(3.2,7.4)$ \\
\hline Time to discharge (min) & $97.9(28.6)$ & $79.0(21.8)$ & 0.02 & $-18.9(-33.4,-4.4)$ \\
\hline $\operatorname{avSS}_{30}$ & $2.4(0.5)$ & $3.5(0.8)$ & $<0.001$ & $1.1(0.7,1.4)$ \\
\hline avVASS $_{30}$ & $1.8(1.6)$ & $3.8(1.7)$ & $<0.001$ & $2.0(1.1,2.9)$ \\
\hline Overall VASS & $2.1(1.7)$ & $4.5(2.5)$ & 0.001 & $2.4(1.1,3.6)$ \\
\hline Av pulse rate first $30 \mathrm{~min}$ (beat $/ \mathrm{min}$ ) & $112.3(16.1)$ & $110.0(20.3)$ & NS & $-2.3(-12.5,7.9)$ \\
\hline Av respiratory rate first $30 \mathrm{~min}\left(\mathrm{~min}^{-1}\right)$ & $28.4(4.9)$ & $25.6(4.8)$ & 0.04 & $-2.9(-5.6,-0.18)$ \\
\hline Av MAPS first $30 \mathrm{~min}(\mathrm{~mm} \mathrm{Hg})$ & $79.7(9.3)$ & $79.0(11.8)$ & NS & $-0.67(-6.7,5.3)$ \\
\hline Av oxygen saturation first $30 \mathrm{~min}(\%)$ & $98.4(0.65)$ & $97.5(3.8)$ & NS & $-0.88(-2.4,0.66)$ \\
\hline
\end{tabular}

$\star$ Intravenous ketamine plus midazolam group. †Intranasal midazolam group. $\neq \mathrm{p}$ Value $>0.05$ considered not statistically significant (NS). \Mean arterial pressure. 


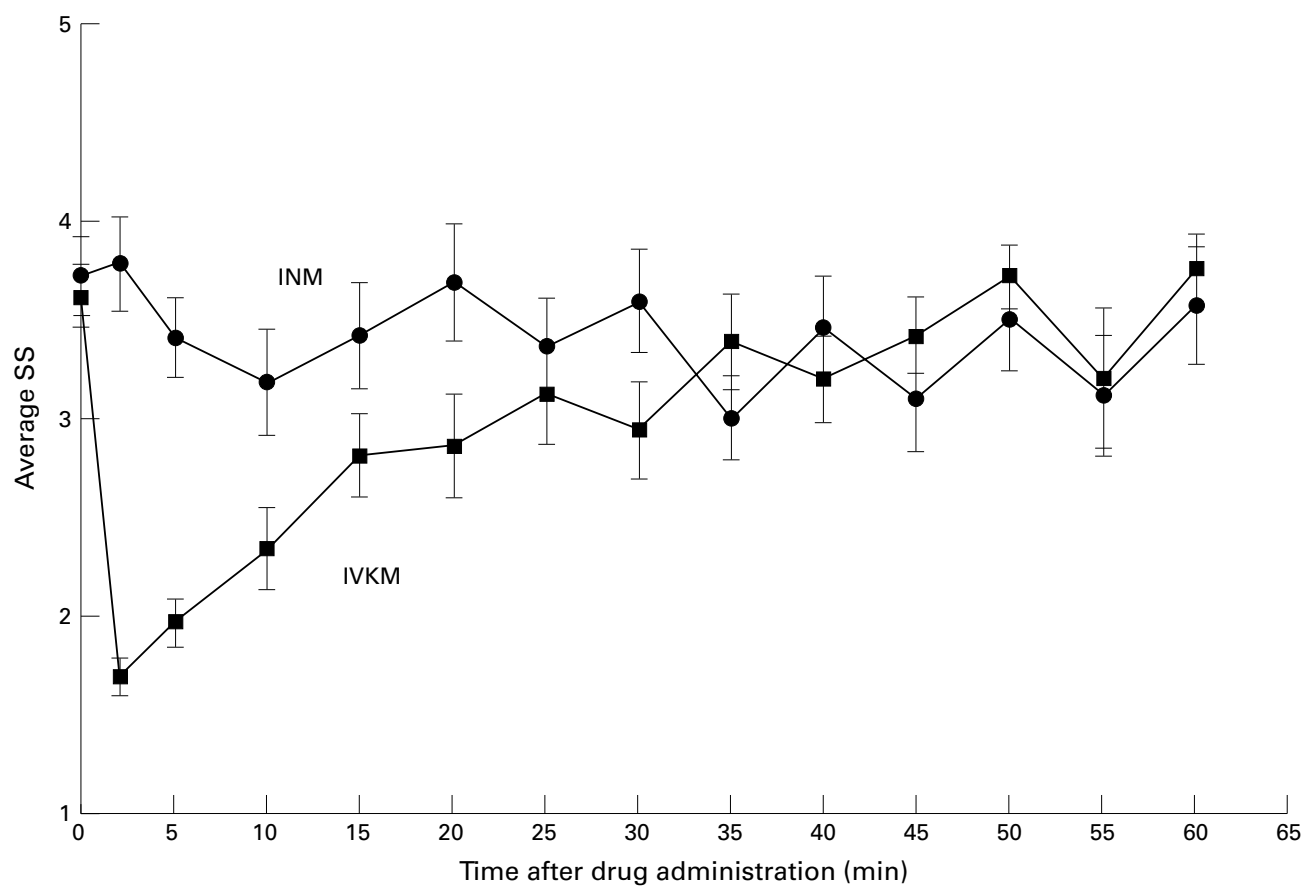

Figure 3 Average Sedation Score (SE) over time after administration for intranasal midazolam (INM) versus intravenous ketamine and midazolam (IVKM).

struggling" (at $10 \mathrm{~cm}$ ). The VASS score corresponded to the distance along the line (measured to the nearest $0.5 \mathrm{~cm}$ ) and was recorded before drug administration then at five minute intervals until the end of the procedure. In addition, at the end of the procedure the doctor recorded a "summary VASS score". Time to onset of sedation was calculated as the time from drug administration until the Sedation Score reached 3 or less. Time to discharge was calculated as the time from drug administration until the child met all the specified criteria for discharge (fig 2). Any adverse events occurring during the procedure or recovery period, as well as any interventions performed, were recorded. Sedation Scores and VASS scores were also averaged over the first 30 minutes after drug administration $\left(\right.$ avSS $_{30}$ and avVASS 30 ). The doctor who performed the procedure and the child's parent were each asked to rate their satisfaction with the sedation technique and if they would want that method of sedation in similar circumstances in the future. Upon discharge, parents were provided with written discharge instructions and asked to complete a brief post-discharge questionnaire to chronicle any adverse events in the week after discharge.

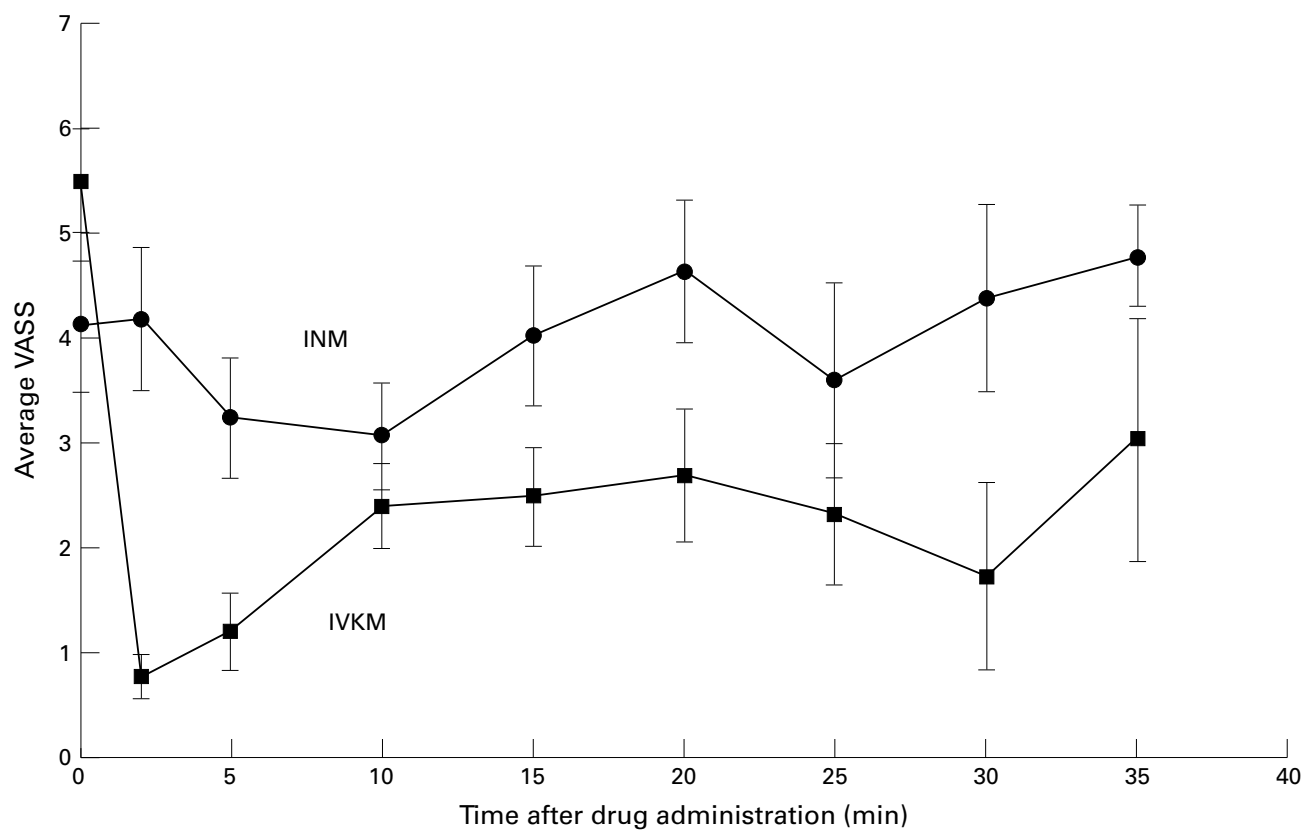

Figure 4 Average Visual Analogue Sedation Scale score (SE) over time after administration for intranasal midazolam (INM) versus intravenous ketamine and midazolam (IVKM). 

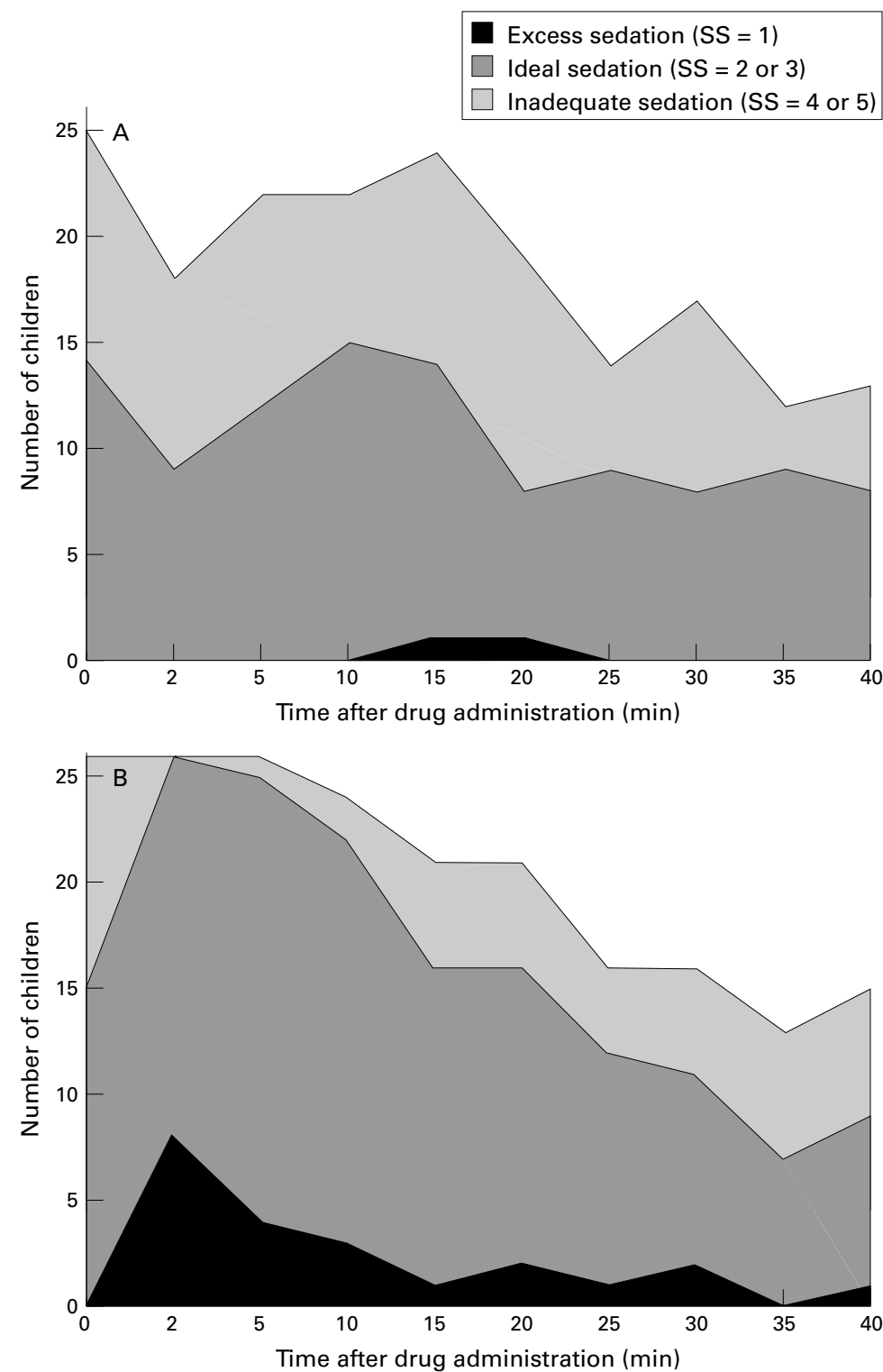

Figure 5 (A) Adequacy of sedation as measured by Sedation Score (SS) across time in children receiving intranasal midazolam (INM). (B) Adequacy of sedation as measured by Sedation Score (SS) across time in children receiving intravenous ketamine and midazolam (IVKM).
Table 3

(A) Doctor assessment of sedation *

\begin{tabular}{lll}
\hline Assessment & $\begin{array}{l}\text { IVKM+ (\%) } \\
n=26\end{array}$ & $\begin{array}{l}I N M \neq(\%) \\
n=26\end{array}$ \\
\hline Excellent & $16(62)$ & $7(27)$ \\
Good & $7(27)$ & $7(27)$ \\
Satisfactory & $3(12)$ & $6(23)$ \\
Poor/Extremely poor & $0(0)$ & $6(23)$ \\
(B) Parent assessment of sedations & \\
Excellent & $17(65)$ & $9(35)$ \\
Good & $7(27)$ & $8(31)$ \\
Satisfactory & $2(8)$ & $4(15)$ \\
Poor/Extremely poor & $0(0)$ & $5(15)$ \\
\end{tabular}

${ }_{\star_{\mathrm{p}} \text { Value }=0.015 \text {. } \dagger \text { Intravenous ketamine plus midazolam }}$ group. $\ddagger$ Intranasal midazolam group. §p Value $=0.042$.

\section{Results}

Fifty three patients were enrolled in the study over a 10 month period. Twenty six of these were randomised to receive INM and 27 to receive IVKM. One patient was inadvertently randomised to the IVKM group before parental consent and was excluded because consent could not be obtained. This left a total of 26 in each group.

There were no significant differences found between the baseline characteristics of the treatment groups with regard to sex, age, weight, procedure type, length or site of laceration, duration of procedure, Sedation Scores or VASS scores, heart rate, blood pressure or oxygen saturation. Mean baseline respiratory rate was however marginally higher in the INM group (table 1), although this difference was not considered to be clinically significant.

All children in the IVKM group were adequately sedated within two minutes of drug administration (table 2) whereas time to onset of sedation in the INM group was more variable (range 2-20 minutes). Children given INM fulfilled the discharge criteria on average 19 minutes earlier (mean 79 minutes, range 45-123 minutes) than those given IVKM (mean 98 minutes, range 50-160 minutes), $\mathrm{p}=0.02$.

Mean avSS mean avVASS $_{30}$ and mean Summary VASS scores were all significantly lower in children given IVKM compared with INM (table 2). Changes in mean Sedation Scores and VASS scores over time are shown in figures 3 and 4 . The number of children in each time period with ideal (Sedation Score 2 or 3), inadequate (Sedation Score $>3$ ) or excess (Sedation Score 1) sedation is shown in figure 5 .

Doctors performing the procedures were more satisfied (table 3A) with the operating conditions given by IVKM than those given by INM, with $88 \%$ rating the sedation as good or excellent for IVKM versus $54 \%$ for INM $(p=0.006)$. In the IVKM group, $95 \%$ of doctors said they would choose that method of sedation again compared with $83 \%$ in the INM group $(p=0.35)$. Parents also reported greater satisfaction (table 3B) with IVKM than INM, with $92 \%$ rating the sedation as good or excellent for IVKM versus $65 \%$ for INM ( $p=0.02)$. In the IVKM group, $95 \%$ of parents said they would choose the same method of sedation again compared with $78 \%$ in the INM group $(\mathrm{p}=0.10)$. 
No serious adverse events were reported in either group. For two children in the INM group the intended procedures could not be completed because of inadequate sedation and general anaesthesia was required. One of these, a 3 year old girl with a foreign body in her ear, was never adequately sedated with INM and remained agitated after a top up dose of intravenous ketamine. One child from the IVKM group required a general anaesthetic after his wound was successfully explored under sedation but then judged to be too extensive for repair in the ED. Another child from the IVKM group required a top up dose of ketamine (at 55 minutes) after the procedure became prolonged. This same child had a brief fall in his oxygen saturation to less than $90 \%$ before the start of the procedure that resolved with jaw support and oxygen administration for one minute. No children in either group experienced laryngospasm or emergence phenomena. Two thirds of the children in the IVKM group were noted to have random movements of the limbs during the procedure but in no case did this interfere with the procedure and only gentle restraint was needed. In two of the INM group there was doubt that the full dose of the study drug was given as some leaked out of the nose but in both cases the sedation was successful. There were no major adverse events reported on the post discharge adverse event records in the week after discharge in the patients for whom these were returned (table 4). Of the listed adverse events, disturbed sleep and increased irritability in the week after the sedation were most frequently reported, but whether or not these were medication induced is uncertain.

When asked to indicate which drug they believed had been given, doctors performing the procedures answered correctly in $55 \%$ of cases (11 of 20) while nurses answered correctly in $70 \%$ of cases ( 14 of 20 ). A total of 36 different doctors and 17 different nurses

Table 4 Adverse events

\begin{tabular}{llll}
\hline Event & $\begin{array}{l}I V K M^{*} \\
(\%)\end{array}$ & $\begin{array}{l}I N M+ \\
(\%)\end{array}$ & $p \neq$ \\
\hline In hospital & $\mathrm{n}=26$ & $\mathrm{n}=26$ & \\
Need for "top up" sedation & 1 & 1 & NS \\
Need for general anaesthesia & 1 & 2 & NS \\
Failed procedure & 0 & 2 & NS \\
Full drug dose not given & 0 & 2 & NS \\
Apnoea/desaturation & 1 & 0 & NS \\
Stridor or laryngospasm & 0 & 0 & NS \\
Vomit during procedure & 0 & 1 & NS \\
Vomit post procedure & 2 & 0 & NS \\
Muscle rigidity & 0 & 0 & NS \\
Random movements & 17 & 0 & $<0.001$ \\
Rash & 1 & 0 & NS \\
Excessive salivation & 1 & 0 & NS \\
Emergence phenomena & 0 & 0 & NS \\
In week after sedation & $\mathrm{n}=19$ & $\mathrm{n}=22$ & \\
Vomiting & 2 & 1 & NS \\
Excessive drooling & 3 & 2 & NS \\
Poor appetite & 3 & 3 & NS \\
Bed wetting & 4 & 1 & NS \\
Irritability & 4 & 5 & NS \\
Disobedience & 6 & 1 & NS \\
Disturbed sleep & 5 & 7 & NS \\
Nightmares & 4 & 2 & NS \\
& & &
\end{tabular}

*Intravenous ketamine plus midazolam group. †Intranasal midazolam group. $\neq$ p Value $>0.05$ considered not statistically significant (NS). were involved in the 52 procedures carried out in this study.

\section{Discussion}

These data demonstrate that both INM and IVKM provide safe and effective sedation for ED minor procedures in children. Sedation was sufficient to complete the procedures in all children receiving IVKM and in $92 \%$ of those receiving INM, however IVKM provided a faster, more predictable sedative response. IVKM administration resulted in a mean Sedation Score (2.5) consistent with a level of sedation previously described as "conscious sedation" ("a state of depressed consciousness that allows protective reflexes to be maintained ... and permits appropriate response by the patient to physical stimulation or verbal command"). The mean Sedation Score in the INM group (3.5) on the other hand, is more consistent with a state between calm and mild agitation. The superior sedation provided by IVKM however also resulted in more children progressing to a deeper level of sedation than desired, a Sedation Score of 1.

Reports of outpatient ketamine use date back to the 1970 s. $^{9}$ Dachs and Innes first published on its use intravenously in the paediatric ED setting in $1997 .{ }^{10}$ In their case series of 30 children given intravenous ketamine (1-2 $\mathrm{mg} / \mathrm{kg}$ ) all were adequately sedated within two minutes. This consistency of effect was confirmed in our study. Children in their study were discharged at a mean time of 25 minutes after drug administration compared with a mean time to discharge in our IVKM group of almost 100 minutes. The addition of intravenous midazolam to ketamine to diminish the risk of emergence phenomena (unpleasant dreams, hallucinations) is known to prolong the sedative effect. ${ }^{11}$ As our department had routinely given midazolam when using intravenous ketamine, we elected to continue the practice for this study. Also, our discharge criteria were more conservative than those of Dachs and Innes, requiring that in addition to a return to a normal conscious level and appropriate verbalisation, children needed to be free of nystagmus and ataxia. Dachs and Innes, in allowing children home earlier, reported post-discharge ataxia that lasted up to two hours in $30 \%$ of their patients. ${ }^{10}$

Despite concerns about the invasiveness of intravenous cannulation, IVKM was found to be highly acceptable to both doctors and parents, a finding consistent with other studies of ketamine use in the paediatric ED. ${ }^{10}{ }^{12-14}$ This study was of insufficient size to evaluate the risk of serious but infrequent side effects of the study medications. An extensive review of ketamine use in children involving 11589 paediatric administrations of the drug demonstrated a high degree of safety. ${ }^{9}$ No children in the IVKM group in our study experienced emergence phenomena. A recent report ${ }^{15}$ has suggested that the incidence of moderate to severe emergence phenomena associated with ketamine use is only $1.6 \%$, considerably less than the previously described rate of up to $10 \% .{ }^{9}$ Hence omission of the midazolam from 
the ketamine protocol, or a reduction of its dose, may allow a faster recovery time without compromising the safety or effectiveness of the sedation.

INM has been shown previously to be an effective means of pre-operative sedation in children. ${ }^{71617}$ Midazolam given via nasal drops (in doses ranging from $0.2-0.5 \mathrm{mg} / \mathrm{kg}$ ) has been shown to be effective in relieving anxiety and facilitating repair of lacerations in children. ${ }^{18-20}$ The onset characteristics and discharge times in our study are consistent with those found in previous trials. ${ }^{7181921}$ Children in the INM group were ready for discharge 19 minutes (on average) earlier than those given IVKM. The economic advantage of this small difference is debatable. Nasal irritation by midazolam may cause distress $(>70 \%$ of children cry after intranasal midazolam ${ }^{17}{ }^{22}$ ) and this may result in administration difficulties. ${ }^{192}$ In our study, INM was given a lower acceptability rating by both doctors and parents. The perception that INM is easier to give and less distressing to children than intravenous cannulation is questionable. Even if true, this slight advantage is negated by its significantly lower efficacy compared with intravenous ketamine. The acidity of the midazolam preparation is probably the main cause of nasal irritation but attempts to change the $\mathrm{pH}$ have been unsuccessful and may change drug absorption. ${ }^{21}$ Nasal spray delivery of midazolam may be less distressing than the common technique of drop instillation. ${ }^{23}$ Further studies investigating the acceptability of intranasal sedative medications in children are needed.

It is possible that despite attempts to blind scorers, the clinical effects of ketamine (nystagmus, random movements, salivation, tachycardia) made obvious which drug had been given. This was not supported by the frequency with which scorers were able to correctly differentiate which form of sedation had been given. Most of the doctors performing the procedures were junior medical officers, usually one to two years postgraduation, had spent fewer than 10 weeks working in our department and had no previous experience with the use of ketamine. The nurses involved in this study were a mix of experienced and inexperienced practitioners. Limited familiarity with the sedative techniques used may have assisted in decreasing evaluator bias.

All of the procedures in this study were performed within the guidelines for paediatric outpatient sedation published by the American Academy of Pediatrics ${ }^{8}$ and the American College of Emergency Physicians. ${ }^{3}$ The use of drug combinations of benzodiazepine with ketamine or an opioid by non-anaesthetists outside of the operating room has been a topic of debate. We would suggest that such drug combinations only be used within agreed guidelines established within individual departments and in communication with the hospital's anaesthesia service. Our guidelines required that an experienced doctor be present, in addition to the doctor performing the procedure, to administer the sedation and be responsible for the safety of the patient. Despite the safety record of the sedation techniques demonstrated in this and other studies, we maintain that these extra safety measures are essential to ensure appropriate management in the event of rare but serious adverse events.

\section{Conclusion}

IVKM used in an appropriate setting by experienced personnel provides an excellent means of achieving a level of sedation suitable for performing most non-painful minor procedures for children in the emergency department. This combination is superior to INM in terms of speed of onset and consistency of effect. INM delivered via an aerosol spray device has a more variable effect but may still be adequate for the completion of many of these procedures.

The authors thank the nursing and registrar staff of the RCH emergency department for their support.

Contributors

Jason Acworth initiated the research, formulated the study hypotheses, designed the protocol, participated in data collection and analysis and wrote the paper. He will act as guarantor for the paper. David Purdie participated in protocol design and the writing of the paper and performed the statistical analysis. Ron Clark initiated the research, discussed core ideas, contributed to protocol development and edited the paper.

Funding: the authors gratefully acknowledge the Royal Children's Hospital Foundation and the Cressbrook Committee for funding this study.

Conflicts of interest: none.

1 Sacchetti A, Schafermeyer R, Gerardi M, et al. Pediatric

analgesia and sedation. Ann Emerg Med 1994;23:237-50.
Andrews JS. Conscious sedation in the pediatric emergency Andrews JS. Conscious sedation in the pediatric
department. Curr Opin Pediatr 1995;7:309-13.

3 American College of Emergency Physicians. Clinical policy for procedural sedation and analgesia in the emergency department. Ann Emerg Med 1998;31:663-77.

4 Cote CJ. Sedation for the pediatric patient-a review. Pediatr Clin North Am 1994;41:31-58.

5 Proudfoot J. Analgesia, anesthesia, and conscious sedation. Emerg Med Clin North Am 1995;13:357-79.

6 O’Neil G, Paech M, Woods F. Preliminary clinical use of a patient-controlled intranasal analgesia (PCINA) device. Anaesth Intens Care 1997;25:408-12.

7 Wilton NCT, Leigh J, Rosen DR, et al. Preanesthetic sedation of preschool children using intranasal midazolam. Anesthesiology 1988;69:972-5.

8 American Academy of Pediatrics, Committee on Drugs. Guidelines for monitoring and management of pediatric Guidelines for monitoring and management of pediatric
patients during and after sedation for diagnostic and therapatients during and after sedation for diagnostic
peutic procedures. Pediatrics 1992;89:1110-15.

peutic procedures. Pediatrics $1992 ; 89: 1110-15$.
9 Green SM, Johnson NE. Ketamine sedation for pediatric procedures: part 2, review and implications. Ann Emerg Med 1990;19:1033-46.

10 Dachs RJ, Innes GM. Intravenous ketamine sedation of pediatric patients in the emergency department. Ann Emerg Med 1997;29:146-50.

11 Reich DL, Silvay G. Ketamine: an update on the first twenty-five years of clinical experience. Can $\mathcal{F}$ Anaesth 1989;36:186-97.

12 Petrack EM, Marx CM, Wright MS. Intramuscular ketamine is superior to meperidine, promethazine, and chlorpromazine for pediatric emergency department sedation. Arch Pediatr Adolesc Med 1996;150:676-81.

13 Green SM, Nakamura R, Johnson NE. Ketamine sedation for pediatric procedures: part 1, a prospective series. Ann Emerg Med 1990;19:1024-32.

14 Parker RJ, Mahan RA, Giugliano D, et al. Efficacy and safety of intravenous midazolam and ketamine as sedation for therapeutic and diagnostic procedures in children. Pediatrics 1997;99:427-31.

15 Green SM, Rothrock RG, Lynch EL, et al. Intramuscular ketamine for pediatric sedation in the emergency department: safety profile in 1022 cases. Ann Emerg Med 1998;31:688-97.

16 Davis PJ, Tome JA, McGowan FX, et al. Preanesthetic medication with intranasal midazolam for brief pediatric surgical procedures. Anesthesiology 1995;82:2-5.

17 Zedie N, Amory DW, Wagner BK, et al. Comparison of intranasal midazolam and sufentanil premedication in pediatric outpatients. Clin Pharmacol Ther 1996;59:341-8.

18 Theroux MC, West DW, Corddry DH, et al. Efficacy of intranasal midazolam in facilitating suturing of lacerations in preschool children in the emergency department. Pediatrics 1993;91:624-7.

19 Connors K, Terndrup TE. Nasal versus oral midazolam for sedation of anxious children undergoing laceration repair. Ann Emerg Med 1994;24:1074-9. 
20 Yealy DM, Ellis JH, Hobbs GD, et al. Intranasal midazolam as a sedative for children during laceration repair. $A m \mathcal{F}$

21 McGlone RG, Ranasinghe S, Durham S. An alternative to "brutacaine": a comparison of low dose intramuscular ketamine with intranasal midazolam in children before suturing. If Accid Emerg Med 1998;15:2316.
22 Karl HW, Rosenberger JL, Larach MG, et al. Transmucosal administration of midazolam for premedication of pediatric patients: comparison of the nasal and sublingual routes. Anesthesiology 1993;78:885-91.

23 Fishbein M, Lugo RA, Woodland J, et al. Evaluation of intranasal midazolam in children undergoing esophagogastroduodenoscopy. I Pediatr Gastroenterol Nutr 1997;25: 261-6. 